Pontifícia Universidade Católica

DO RIO DE JANEIRO

Sergio Ibajé Oliveira Bueno

\title{
AVALIAÇÃO ESTRUTURAL DE DUTOS COM DEFEITOS DE CORROSÃO COINCIDENTES COM A SOLDA LONGITUDINAL
}

Dissertação de Mestrado

Dissertação apresentada ao Programa de Pós-graduação em Engenharia Mecânica da PUC-Rio como requisito parcial para obtenção do título de Mestre em Ciências de Engenharia Mecânica.

Orientador: José Luiz de França Freire Co-orientador: Adilson Carvalho Benjamin 
Pontifícia Universidade Católica

DO RIO DE JANEIRO

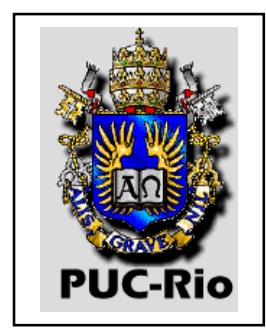

\title{
Sergio Ibajé Oliveira Bueno
}

\section{Avaliação Estrutural de Dutos com Defeitos de Corrosão Coincidentes com a Solda Longitudinal}

\begin{abstract}
Dissertação apresentada como requisito parcial para obtenção do grau de Mestre pelo Programa de Pósgraduação em Engenharia Mecânica da PUC-Rio. Aprovada pela Comissão Examinadora abaixo assinada.
\end{abstract}

Prof. José Luiz de França Freire Orientador Departamento de Engenharia Mecânica - PUC-Rio

Prof. Ronaldo Domingues Vieira Departamento de Engenharia Mecânica - PUC-Rio

Prof. Valter Rocha dos Santos Departamento de Engenharia Metalúrgica - PUC-Rio

Dr. Adilson Carvalho Benjamin Petrobras/Cenpes Dr. Alexandre Meirelles Pope Petrobras/Cenpes Prof. José Eugênio Leal Coordenador Setorial do Centro Técnico Científico Rio de Janeiro, 02 de maio de 2007. 
Todos os direitos reservados. É proibida a reprodução total ou parcial do trabalho sem autorização da universidade, do autor e do orientador.

\section{Sergio Ibajé Oliveira Bueno}

Graduou-se em Engenharia Metalúrgica na UFF (Universidade Federal Fluminense) em 1987. Cursou Engenharia de Equipamentos (ênfase Inspeção) na Petrobras em 1989 e Engenharia de Dutos na PUC-Rio em 2001. Atuou nas áreas de inspeção de equipamentos de plataformas e avaliação de integridade de dutos e equipamentos submarinos. Participou de cursos e congressos, no Brasil e no exterior, na área de engenharia de dutos. É responsável pela análise de integridade estrutural de dutos e equipamentos submarinos na PETROBRAS/Unidade de Negócios Bacia de Campos/Suporte Técnico.

Ficha Catalográfica

Bueno, Sergio Ibajé Oliveira

Avaliação estrutural de dutos com defeitos de corrosão coincidentes com a Solda Longitudinal / Sergio Ibajé Oliveira Bueno; orientador: José Luiz de França; co-orientador: Adilson Carvalho Benjamin. - Rio de Janeiro : PUC, Departamento de Engenharia Mecânica, 2007.

[17], 202 f. : il. ; $30 \mathrm{~cm}$

Dissertação (mestrado) - Pontifícia Universidade Católica do Rio de Janeiro, Departamento de Engenharia Mecânica.

Inclui referências bibliográficas.

1. Engenharia mecânica - Teses. 2. Dutos. 3. Defeitos de corrosão. 4. Solda Longitudinal. 5. Avaliação estrutural. 6. Métodos de avaliação. I. Freire, José Luiz de França. II. Benjamin, Adilson Carvalho. III. Pontifícia Universidade Católica do Rio de Janeiro. Departamento de Engenharia Mecânica. IV. Título. 
Dedico esta dissertação aos trabalhadores do Brasil, em especial aqueles que dedicam sua vida na exploração e produção de petróleo nas plataformas da Bacia de Campos. Trabalhadores que com sua luta diária e muitas vezes pouco reconhecida garantem a produção de energia tão necessária à sociedade brasileira.

Com base no vosso exemplo procurei fazer o melhor. 


\section{Agradecimentos}

Ao Professor José Luiz de França Freire, pela orientação e aprendizado durante o curso de Engenharia de Dutos e na elaboração da tese.

Ao meu Co-orientador Adilson Carvalho Benjamin, pela paciência, generosidade em transferir conhecimentos adquiridos em vários anos de pesquisa, pela orientação e oportunidade de desenvolver este trabalho ao seu lado.

Ao professor Ronaldo Domingues Vieira, pela atenção durante a realização dos ensaios e discussão dos resultados.

Ao amigo Marcelo Torres Piza Paes pela orientação e diversas correções nos aspectos relacionados a materiais, bem como pelo incentivo e apoio se mostrando sempre a disposição apesar dos seus diversos afazeres e viagens.

Aos meus pais Adão e Cely que sempre me incentivaram nos estudos me deixando a vontade para traçar meu caminho.

À minha mulher Liliane que com seu carinho e compreensão soube me apoiar nos momentos difíceis e tornar este período o mais leve possível.

Ao meu filho João Pedro, simplesmente por ele existir e ser para mim um motivo de muita felicidade.

Á minha equipe de trabalho composta pelos Engenheiros Aldo Renato Franzoi, Ricardo Pereira de Morais, Rodolfo José de Moura Neto, Carlos Eduardo Amosso Medeiros e pelos Técnicos de Inspeção Francimário da Silva Vieira de Melo, Jussara Carvalho Ribeiro e Esmir Gonçalves pelo incentivo e por terem suprido minha ausência necessária para a produção desta dissertação. 
Aos meus Gerentes na PETROBRAS Sergio Fonseca Candido e Guilherme de Almeida Peixoto que souberam compreender a necessidade de imersão e dedicação exclusiva para cursar as disciplinas e elaborar a dissertação.

Aos amigos do CENTRO de PESQUISAS da PETROBRAS, Rodrigo Hoppe, José Cláudio Guimarães Teixeira e João Luís do Nascimento que me ajudaram no entendimento dos fenômenos da Tese relacionados às propriedades mecânicas do material.

Ao Engenheiro Ricardo Dias de Souza pela ajuda inicial com dados dos tubos do ORBEL e pelo trabalho prévio tantas vezes citado nesta Tese.

Às secretarias da Gerência de Elevação e Escoamento da UN-BC Aline Sodero Lemos e Ana Cristina de Oliveira pela ajuda dedicada na preparação das viagens.

Aos amigos, colegas de mestrado da PUC-RIO, Leonardo Dantas Rodrigues, Marco Antonio Pérez Rosas, Habib Zambrano e José de Jesús Leal Carvajalino pela acolhida e pelos bons momentos vividos.

Aos Técnicos de Metalografia da PUC-RIO Marco Aurélio Pestana Guimarães e Heitor Nuss Guimarães pela ajuda na preparação e análise das microestruturas.

Ao Dr. Marcos Henrique de Pinho Maurício do Departamento de Ciência dos Materiais e Metalurgia da PUC-RIO pela ajuda nas avaliações no MEV e nas medições de tamanhos de grão.

Aos professores que participaram da Comissão examinadora.

A todos que não foram citados acima, porém que tiveram participação na elaboração da minha tese. 


\section{Resumo}

Bueno, Sergio Ibajé Oliveira; Freire, José Luiz de França (orientador). Avaliação Estrutural de Dutos com Defeitos de Corrosão Coincidentes com a Solda Longitudinal. Rio de Janeiro, 2007, 202p. Dissertação de Mestrado - Departamento de Engenharia Mecânica, Pontifícia Universidade Católica do Rio de Janeiro.

A redução de espessura de parede causada por corrosão é um dos defeitos que mais afetam a integridade dos dutos. Estes defeitos podem ocorrer no metal base, nas soldas longitudinais ou circunferenciais, bem como nas zonas afetadas pelo calor. Os métodos de avaliação da resistência remanescente introduzem ressalvas ou proíbem o tratamento de defeitos de corrosão coincidentes com as juntas soldadas. No presente trabalho foram avaliados os níveis de segurança dos métodos usuais de avaliação da resistência de dutos com defeitos introduzidos na região da solda longitudinal de tubos soldados por arco submerso. Os tubos testados eram de aço C-Mn fabricados na década de 60 e foram retirados de operação após uma campanha superior a 30 anos. Com estes tubos foram fabricados 5 espécimes, cada qual com 1 defeito externo produzido por eletro-erosão, sendo posteriormente submetidos a testes de pressão monitorados. Foram realizados ensaios de tração, ensaios de impacto Charpy e ensaios de dobramento, para determinação das propriedades mecânicas do metal de base, do metal de solda e da zona termicamente afetada (ZTA). Foram realizados também ensaios metalográficos do metal de base e do metal de solda, análise química do metal de base e do metal de solda e medição das tensões residuais no metal de solda. A aplicação dos métodos de nível 1 (ASME B31G, 085dL, RPA, DNV isolado e PCORRC) resultou em pressões previstas inferiores às pressões reais de ruptura para defeitos de formato esférico. Para defeitos de seção retangular a aplicação dos métodos de nível 1 resultou em pressões superiores às pressões reais. A aplicação dos métodos de nível 2 (Effective Area e DNV geometria complexa) resultou em pressões previstas superiores às pressões reais de ruptura para defeitos de formato esférico e de seção retangular. As análises das fraturas indicaram que não ocorreu falha por deficiência de tenacidade em nenhum espécime. A corrosão alveolar leve pré-existente na superfície interna dos espécimes foi apontada 
como causa mais provável das pressões previstas superiores às pressões reais de ruptura.

\section{Palavras-chave}

Dutos; defeitos de corrosão; solda longitudinal; avaliação estrutural; métodos de avaliação. 


\section{Abstract}

Bueno, Sergio Ibajé Oliveira; Freire, José Luiz de França (advisor). Structural Assessment of Pipelines with Blunt Corrosion Defects Coincident with Long-Seam Welds. Rio de Janeiro, 2007, 202p. Masters Thesis - Department of Mechanics, Pontifical Catholic University of Rio de Janeiro.

One of the most important issues that affects pipeline integrity is corrosioncaused metal loss. This type of defect can occur over the pipe body, seam or girth welds or even on heat affected zones. Pipeline remaining strength criterions are restricted or even prohibited for assessing corrosion defects coincident with weld regions. This present work investigated the reliability of the most common assessment criteria for corroded pipes when defects were coincident with seam weld region in submerged arc welded pipes. The tested specimens were C-Mn steel pipe, from a pipeline manufactured in the 60's and had operated for over 30 years. Five external defects were created on five pipe specimens and pressure tested until rupture. Stress, charpy V-notch and bending tests were carried out to find the mechanical properties of the welded joints. Microstructure and chemical composition from base metal and weld metal were also analyzed. Residual stresses on welds were measured. The level 1 criterion (ASME B31G, 085dL, RPA, DNV single and PCORRC) applications have underestimated failure pressure for spherical shaped defects. On the other hand, the same criterions have overestimated failure pressure for rectangular shaped defects. The level 2 criterions (Effective Area e DNV complex geometry) application overestimated failure pressure for all 5 specimens with both spherical and rectangular shaped defects. Fracture analyses have indicated that no specimen had a toughness controlled failure. Internal pit corrosion that was found after pressure testing is recognized as the most probable cause of underestimated pressure forecasts. 


\section{Keywords:}

Pipelines; corrosion defects; long-seam welds; structural assessment; assessment methods. 


\section{Sumário}

LISTA DE SIGLAS 23

1 Introdução 24

1.1. Evolução dos Métodos de Avaliação de Integridade de Dutos na

Presença de Defeitos de Corrosão 25

1.2. Evolução dos Métodos de Avaliação de Integridade de Dutos na

Presença de Defeitos de Corrosão na PETROBRAS e na PUC-RIO 28

1.3. Motivação do tema da dissertação 29

1.4. Apresentação dos próximos capítulos desta Dissertação 30

2 Revisão da Literatura 33

2.1. Aspectos de Corrosão em Dutos 34

2.1.1. Aspectos gerais da corrosão interna de dutos 35

2.1.2. Corrosão pela ação de microrganismos em dutos submarinos 35

2.1.3. Corrosão pelo $\mathrm{H}_{2} \mathrm{~S}$ e $\mathrm{CO}_{2}$

2.1.4. Possibilidade das Soldas Longitudinais serem atingidas pela corrosão 39

2.1.5. Corrosão Preferencial na Solda 41

2.2. Aços utilizados na fabricação de tubos 43

2.2.1. Principais normas de especificação de materiais para tubos 45

2.3. Processos de soldagem na fabricação de tubos 46

2.3.1. Soldagem Arco Submerso (SAW) e Conformação UOE 47

2.3.2. A velocidade de resfriamento em processo de soldagem SAW 50

2.3.3. Soldagem por Resistência Elétrica (ERW) 52

2.4. Fabricação de Tubos sem Costura 55

2.5. Restrições à avaliação da resistência de dutos com defeitos em soldas 56

2.6. Aspectos de Tenacidade relacionados aos Métodos de Avaliação de Resistência Remanescente de dutos com Defeitos de Corrosão. 61

2.7. Requisitos de Tenacidade (RT) da ZTA das Normas de Projeto 63

2.8. Dificuldades na Avaliação da Tenacidade da ZTA em tubos SAW 64

2.9. Princípios de Análise de Fratura 65

2.10. Influência da espessura na tenacidade da junta soldada 67

2.11. Avaliação de Tensões Residuais pela Técnica do Furo Cego 69 
$\begin{array}{ll}3 \text { Procedimento experimental } & 72\end{array}$

3.1. Origem dos Segmentos de Tubos 72

3.1.1. Histórico Operacional do Oleoduto que deu Origem aos Segmentos $\begin{array}{ll}\text { de Tubos } & 73\end{array}$

3.1.2. Seleção e identificação dos segmentos de tubos 73

3.2. Qualificação do material 75

3.2.1. Ensaios de Tração $\quad 75$

3.2.1.1. Metodologia de Ensaio $\quad 76$

3.2.2. Ensaios de Dobramento $\quad 79$

3.2.3. Ensaios de Impacto com CPs de dimensões reduzidas 80

3.2.4. Avaliação das Tensões Residuais pela Técnica do "Furo Cego" 80

3.2.5. Análise Química do Metal Base e do Metal de Solda 81

3.2.6. Análise Microestrutural e Medição de Microdureza 81

3.3. Testes de pressão interna ou de ruptura 81

3.3.1. Fechamento dos Espécimes Tubulares 82

3.3.2. Fabricação dos Defeitos

3.3.2.1. Considerações sobre a Usinagem dos Rebaixos Esféricos 83

3.3.2.2. Considerações sobre a Usinagem dos Rebaixos Retangulares $\quad 85$

3.3.2.3. Levantamento Dimensional dos Espécimes Tubulares 86

3.3.3. Testes de Pressão nos Espécimes Tubulares $\quad 87$

3.3.3.1. Medições de Deformação, Pressão e Volume Injetado 89

3.3.3.2. Medições de Deslocamento Radial no Centro dos Defeitos 89

3.3.3.3. Condicionamento dos Sinais 90

3.3.3.4. Registro em Vídeo dos Testes 90

$\begin{array}{ll}\text { 3.3.3.5. Incertezas das Medidas } & 91\end{array}$

4 Resultados Obtidos nos Ensaios $\quad 92$

4.1. Ensaios de Tração 92

4.2. Ensaios de Dobramento 96

4.3. Análise Química do Metal Base e do Metal de Solda 96

4.4. Medição de microdureza $\quad 97$

4.5. Análise Microestrutural $\quad 100$

4.6. Ensaios de Impacto com CPs de dimensões reduzidas 105

4.7. Tensões Residuais pela Técnica do "Furo Cego" 110

4.8. Resultados do Levantamento Dimensional dos Espécimes Tubulares 113

$\begin{array}{ll}\text { 4.9. Testes de ruptura } & 115\end{array}$ 
4.10. Comparação entre as Deformações Medidas pelos Strain Gages (SG) 121

5 Discussão dos Resultados dos Testes de Ruptura 127

5.1. Análise do Perfil de Corrosão Interna dos ETs 127

5.1.1. Resultados das pressões previstas de ruptura pelos métodos de nível 1 considerando a contribuição dos alvéolos

5.1.2. Resultados das pressões previstas de ruptura pelos métodos de nível 2 considerando a contribuição dos alvéolos

5.2. Comparação com experimento da literatura

5.3. Análise Fratográfica dos locais do vazamento dos ETs retangulares

(15 e 16)

5.4. Análise Fratográfica dos locais do vazamento dos ETs esféricos

$(11,13$ e 14)

5.5. Análise pelo Método dos Elementos Finitos da Intensificação de Tensões nos ETs retangulares.

6 Conclusões

7 Recomendações de Trabalhos Futuros

8 Referências Bibliográficas

APÊNDICE A - Valores Medidos nos Ensaios de Tração 158

CPs TRANSVERSAIS CONTENDO A SOLDA LONGITUDINAL 158

CPS LONGITUDINAIS RETIRADOS APENAS DO METAL DE SOLDA 159

CPS LONGITUDINAIS RETIRADOS DO METAL BASE 161

CPS TRANSVERSAIS RETIRADOS DO METAL BASE 163

APÊNDICE B - Memória de Cálculo da Resistência Remanescente dos ETs com defeitos pelo Método da API 579

APÊNDICE C - Fotos e Croquis dos ETs após Ruptura com Dimensões dos Alvéolos 
APÊNDICE D - Telas dos Programas RSTRENG e DNV-RP-F101

RSTRENG

DNV-RP-F101

APÊNDICE E - Análise das Superfícies de Fratura dos CPs de Ensaio

Charpy com auxílio do MEV

APÊNDICE F - Análise das Superfícies de Fratura do ET 14 com

auxílio do MEV

APÊNDICE G - Equações dos Métodos de Avaliação da Resistência

Remanescente de Dutos Corroídos

G.1 - Métodos de Nível 1

G.2 - Métodos de Nível 2

190

APÊNDICE H- Estimativa de Tensões atuantes nos defeitos dos ETs retangulares com a influência dos alvéolos

Modelagem da seção transversal sem considerar a presença de alvéolos 


\section{Lista de Figuras}

Figura 2-1 - Aspecto da corrosão induzida por microrganismos na

presença de incrustação de sulfato de bário e estrôncio $\left(\mathrm{BaSO}_{4}, \mathrm{SrSO}_{4}\right)$

Figura 2-2 - Corrosão Dispersa por toda a circunferência do

duto - BRS associada a $\mathrm{BaSO}_{4}$

Figura 2-3 - Posição da Solda Longitudinal em relação à Geratriz

Inferior (faixa branca - indicação 6h)

Figura 2-4 - Corrosão Localizada na Geratriz Inferior - Duto com elevado BSW

Figura 2-5 - Ilustração mostrando alguns itens da solda por arco submerso. 48

Figura 2-6 - Esquema ilustrativo do processo U-O-E de fabricação de tubos. 50

Figura 2-7 - Esquema simplificado do processo ERW com a corrente gerada por eletrodos de contato (esquerda) e por indução (direita)

Figura 2-8 - Ilustração da perfuração do bloco na fabricação de tubos sem costura pelo método rotary piercing

Figura 2-9 - Constrição à deformação devido ao material não tensionado

Figura 2-10- Variação do comportamento do material de dútil para

frágil em função da espessura

Figura 2-11 - llustração da relação dos diâmetros do furo e do local de medição da tensão residual

Figura 2-12 - Desenho esquemático de extensômetros de três elementos (tipo A) usado na Técnica do Furo Cego

Figura 3-1 - Diagrama simplificado do Oleoduto ORBEL

Figura 3-2 - Ilustração mostrando os tipos de CPs ensaiados à tração

Figura 3-3 - Ilustração mostrando as dimensões A (cutelo) e

$B$ (suporte) do ensaio de dobramento

Figura 3-4 - Esquema de orientação para fabricação dos

eletrodos para confecção dos defeitos dos ETs 11, 13 e 14

Figura 3-5 - Seções longitudinais dos defeitos produzidos por eletro-erosão nos ETs 11, 13 e 14 (dimensões em mm)

Figura 3-6 - Seções longitudinais e vista de topo dos defeitos usinados nos ETs 15 e 16 (dimensões em mm)

Figura 3-7 - Esquema mostrando as localizações, direções e identificações 
das rosetas extensométricas (ETs 11, 13 e 14)

Figura 3-8 - Esquema mostrando as localizações, direções e identificações das rosetas extensométricas (ETs 15 e 16)

Figura 3-9 - Ilustração do apalpador utilizado para medir o deslocamento radial da superfície do defeito

Figura 4-1 - Resultados de Limite de escoamento (LE) dos CPs MB Transv (Metal Base na direção Transversal), MB Long (Metal Base da direção Longitudinal) comparado com a Especificação do API 5L para o Limite de Escoamento Transversal (317 MPa)

Figura 4-2 - Resultados de Limite de Resistência à Tração dos CPs MB Transv (Metal Base na direção Transversal), MB Long (Metal Base da direção Longitudinal) comparado com a Especificação do API 5L para a Limite de Resistência à Tração Transversal (434 MPa)

Figura 4-3 - Resultados de Tensão Limite de Escoamento dos CPs T (MB Transv), L (Metal Base Longitudinal) e M (All weld longitudinal retirado apenas do metal de solda)

Figura 4-4 - Resultados de $\sigma_{y} / \sigma_{u}$ na longitudinal (L) e transversal (T) comparados com o limite superior de 0,93 da API 5L [18]

Figura 4-5 - Resultados da razão entre $\sigma_{y} / \sigma_{u}$ na longitudinal (L) e na transversal (T) comparados com o limite superior de 1,02 da DNV OS-F101 [31]

Figura 4-6- Esquema ilustrativo da localização dos pontos de medição de microdureza Vickers na junta soldada

Figura 4-7- Perfil de microdureza das junta soldada dos tubos 13 e 14

Figura 4-8 - Junta soldada mostrando os passes interno (inferior) e

externo (superior) e a ZTA - ataque: nital $2 \%$

Figura 4-9 - Ferrita poligonal e perlita (Tubo 16) - 200X

- ataque: nital $2 \%$

Figura 4-10 - Ferrita poligonal e de aparência acicular (Tubos 15 e 14)

$-200 X$ - ataque: nital $2 \%$

Figura 4-11 - Ferrita com aparência acicular (Tubos 11 e 13) - 200X

- ataque: nital $2 \%$

Figura 4-12 - Metalografia do Tubo 13 apresentando alinhamento microestrutural - 200X - ataque: nital $2 \%$

Figura 4-13 - Caracterização microestrutural do passe interno

(Tubos 11 e 13) - 100X - ataque: nital $2 \%$ 
Figura 4-14 - Caracterização microestrutural dos passes interno, externo e ZTA (Tubo 15) - 100X - ataque: nital $2 \%$

Figura 4-15 - Região de grãos grosseiros da ZTA do Tubo 13 - 200X - ataque: nital $2 \%$

Figura 4-16 - Microestruturas do metal base dos tubos 14, 11 e 16 mostrando o corte transversal de inclusões alongadas - 500X ataque: nital $2 \%$

Figura 4-17 - Valores de Energia absorvida a $-20{ }^{\circ} \mathrm{C}$ no Ensaio Charpy dos CPs com entalhes posicionados em 5 regiões da junta soldada.

Figura 4-18 - Valores de Energia absorvida a $0{ }^{\circ} \mathrm{C}$ no Ensaio Charpy dos CPs com entalhes posicionados em 5 regiões da junta soldada.

Figura 4-19 - Valores de Energia absorvida a $+22{ }^{\circ} \mathrm{C}$ no Ensaio Charpy dos CPs com entalhes posicionados em 5 regiões da junta soldada

Figura 4-20 - Comparação da tenacidade medida no Ensaio

Charpy (Temperatura de $+20^{\circ} \mathrm{C}$ ) de aços da literatura com os obtidos nos CPs dos tubos

Figura 4-21 - Variação da Energia Absorvida (J) por ET em função da temperatura do Ensaio Charpy

Figura 4-22 - Valores das Tensões Residuais medidas nas direções longitudinal e circunferencial em vários pontos da circunferência do ET 16 - Técnica do Furo Cego

Figura 4-23 - Valores das Tensões Residuais Máximas e Mínimas em vários pontos da circunferência do ET 16 - Técnica do Furo Cego

Figura 4-24- Vista de topo do defeito produzido por eletro-erosão no ET 13115 Figura 4-25 - Vista de topo do defeito produzido por eletro-erosão no ET 15

Figura 4-26 - Parâmetros de forma $\left(L^{2} / D t\right)$ dos defeitos dos ETs

Figura 4-27 - Comparação dos resultados da pressão real (Prup) de ruptura (linha vermelha pontilhada) com os pressão previstas (Pmétodo) pelos diversos métodos

Figura 4-28 - Descarregamento local no ponto de instalação das rosetas 7 dos ETs retangulares 15 e 16 comparados com os ETs cilíndricos

Figura 4-29- Comparação do deslocamento radial (abaulamento) do centro do defeitos dos ETs

Figura 4-30 - Descontinuidade de medições indicando o descolamento dos SG 7 e 10 
Figura 5-1 - ET 14 após o teste de pressão com as marcações para o corte. 127

Figura 5-2 - Superfície interna do ET 11 após o corte.

Figura 5-3 - Cálculos da Pressão de Ruptura considerando a perda

de espessura relativa aos alvéolos de corrosão interna

(Métodos de Nível 1)

132

Figura 5-4- Sensibilidade dos Métodos de Nível 2 à presença dos

alvéolos internos de corrosão.

Figura 5-5 - Comparação das características das soldas da referencia

(SSAW-direita) e dos ETs (DSAW-esquerda)

Figura 5-6 - Espécime Tubular para Teste de Pressão mostrando o

segmento contendo os defeitos soldados em tubo íntegro.

Figura 5-7 - Detalhe dos três defeitos simulados de corrosão

produzidos por usinagem

Figura 5-8 - Detalhe dos três defeitos simulados de corrosão produzidos por usinagem

Figura 5-9 - Medições de espessura (em $\mathrm{mm}$ ) realizadas com paquímetro após o teste de ruptura.

Figura 5-10 - Medições de espessura (em mm) realizadas com ultra-som antes do teste de ruptura.

Figura 5-11 - Detalhe do local do vazamento do ET 15

(comprimento: $4 \mathrm{~mm}$, profundidade: $0,8 \mathrm{~mm}$ )

Figura 5-12 - Detalhe do local do vazamento do ET 16 mostrando

o alvéolo interno na parte de baixo e a pequena estricção na parte de cima

Figura 5-13 - Detalhe mostrando o local do vazamento visto ao microscópio ótico (aumento 200X)

Figura 5-14 - Foto do local de vazamento do ET 15 na borda do defeito

Figura 5-15 - Foto do ET 11 mostrando o local do vazamento entre a

solda e o metal base

Figura 5-16 - Análise macroscópica da falha do ET 14 mostrando

detalhe do início da fratura e do rasgamento dútil

Figura 5-17 - Análise macroscópica da falha do ET 14 mostrando

detalhe do início da fratura na superfície interna

Figura 5-18 - Curva Tensão-deformação em um CP da direção

transversal do tubo 16

Figura E-1 - Regiões de clivagem (ET 15; $-20^{\circ} \mathrm{C}$; MB) -

Aumentos 40X e 500X 
Figura E-2 - Parcela da fratura de característica dútil mostrando as

cavidades e raias de rasgamento $\left(\mathrm{ET} 13 ;-20^{\circ} \mathrm{C}\right.$; MB)

Figura E-3 - Inclusão de Mns observada na área dútil do CP

(ET 13; $-20^{\circ} \mathrm{C} ; \mathrm{MB}$ )

Figura E-4 - Inclusão de óxidos $\left(\mathrm{MnO}-\mathrm{SiO}_{2}\right)$ caracterizada pela

análise EDS. (amostra 5)

178

Figura $\mathrm{E}-5$ - Inclusão de óxidos $\left(\mathrm{MnO}-\mathrm{SiO}_{2}\right)$ e sulfetos (MnS)

caracterizada pela análise EDS. (amostra 5 )

Figura E-6 - Inclusão de óxidos $\left(\mathrm{MnO}-\mathrm{SiO}_{2}\right)$ caracterizada pela

análise EDS. (ET $15 ;-20^{\circ} \mathrm{C}$; CS)

Figura E-7 - Inclusão de óxidos $\left(\mathrm{MnO}-\mathrm{SiO}_{2}\right)$ caracterizada pela análise EDS. (ET $14 ; 20^{\circ} \mathrm{C}$; CS)

Figura E-8 - Planos de clivagem associados a microcavidades

(ET 16; $0^{\circ} \mathrm{C} ; \mathrm{LF}+5 \mathrm{~mm}$ )

Figura F-1 - Visualização dos segmentos da fratura a serem analisados no MEV

Figura F-2 - Visualização do bandeamento das amostras A1, B2 e B1

Figura F-3 - Visualização do aspecto dútil da fratura (microcavidades)

no ponto de iniciação - região $A 1$

Figura F-4 - Visualização do aspecto dútil da fratura (microcavidades)

afastado do ponto de iniciação - região A1

186

Figura G-1 - Representação da área longitudinal perdida por meio de uma área parabólica e retangular

Figura G-2 - Detalhe dos comprimentos para o cálculo pelo método

"Effective Area"

Figura G-3 - Subdivisão de um defeito de geometria complexa

em "patch" e "pits"

Figura G-4 - Definição da área de patch $\left(A_{\text {patch }}\right)$ e área de pit

$\left(A_{\text {pit }}\right)$ para subdivisão de uma geometria complexa em "patch"

e "pits" idealizados

Figura G-5 - Exemplo de agrupamento de defeitos adjacentes para interação

Figura G-6 - Combinação de defeitos em interação

Figura H-1 - Simulação pelo MEF da seção transversal do defeito

sem a influência de alvéolos

Figura H-2 - Plastificação do ligamento na presença de um alvéolo 
interno com tensão constante em toda a seção de aproximadamente $548 \mathrm{MPa}$ para um carregamento de $41 \mathrm{kgf} / \mathrm{cm}^{2}$ de pressão

Figura H-3 - Plastificação do ligamento na presença de três alvéolo interno com tensão máxima de aproximadamente 548 MPa para um carregamento de $39 \mathrm{kgf} / \mathrm{cm}^{2}$ de pressão

Figura H-4 - Detalhe da plastificação do ligamento na presença de três alvéolos internos para um carregamento de $39 \mathrm{kgf} / \mathrm{cm}^{2}$ de pressão 


\section{Lista de Tabelas}

Tabela 2-2 - Composição química de aço X80 para a fabricação de tubos ERW.

Tabela 3-1 - Exemplo de pressões $\left[\mathrm{kgf} / \mathrm{cm}^{2}\right]$ aplicadas em teste hidrostático no ORBEL I.

Tabela 3-2- Resumo da Inspeção realizada no canteiro após o recebimento dos tubos

Tabela 3-3- Posição dos tubos retirados do Oleoduto

Tabela 3-4 - Dimensões dos defeitos conforme projetado -

ETs 11,13 e 14

Tabela 4-1 - Resultados das Análises Químicas do Metal Base (espectrometria por emissão ótica ) e Comparação com especificações API 5L e DNV OS F-101 atuais

Tabela 4-2 - Resultados de microdureza das Juntas Soldadas amostradas dos segmentos de tubos.

Tabela 4-3 - Resultados de Medições das Tensões Residuais realizadas no centro da solda longitudinal pela Técnica do Furo Cego.

Tabela 4-4 - Resumo das medições realizadas nos ETs após a introdução dos defeitos.

Tabela 4-5 - Pressões de Falha dos ETs sem defeitos pelos diversos métodos de instabilidade $\left(\mathrm{kgf} / \mathrm{cm}^{2}\right)$.

Tabela 4-6 - Resultados de pressões calculadas pelos métodos ( $\left.P_{\text {método }}\right)$

e das pressões obtidas $\left(P_{\text {rup }}\right)$ nos testes de ruptura $\left(\mathrm{kgf} / \mathrm{cm}^{2}\right)$

Tabela 4-7 - Erros Percentuais dos Métodos na Previsão das

Pressões de Ruptura

Tabela 4-8 - Deformações circunferenciais máximas medidas nos

ETs com defeitos esféricos.

Tabela 5-1 - Resumo da contribuição dos alvéolos e da limitação dos métodos

Tabela 5-2 - Alteração das relações d/t devido a consideração da contribuição dos alvéolos internos

Tabela 5-3 - Resultados de pressões calculadas pelos métodos e das pressões obtidas nos testes de ruptura $\left(\mathrm{kgf} / \mathrm{cm}^{2}\right)$ considerando 
a influência dos alvéolos.

Tabela 5-4 - Erros Percentuais dos Métodos de Nível 1 considerando a influência dos alvéolos na Previsão das Pressões de Ruptura

Tabela 5-5 - Resultados de pressões calculadas pelos métodos e das pressões obtidas nos testes de ruptura $\left(\mathrm{kgf} / \mathrm{cm}^{2}\right)$ considerando a influência dos alvéolos.

Tabela 5-6 - Erros Percentuais dos Métodos de Nível 2 considerando a influência dos alvéolos na Previsão das Pressões de Ruptura

Tabela 5-7 - Dados Técnicos de Tubos antigos testados com defeitos usinados englobando a solda longitudinal

Tabela 5-8 - Dimensões dos defeitos projetados para serem usinados

Tabela 5-9- Resultados dos Testes de Pressão dos Defeitos Usinados

Tabela E-1 - Características das amostras selecionadas para análise no MEV 


\section{LISTA DE SIGLAS}

BRS - Bactéria Redutora de Sulfato.

BSW - Basic Sediments and Water (Teor de Água e Sedimentos)

DEM-PUC-Rio - Departamento de Engenharia Mecânica da PUC-Rio.

DNV - Det Norske Veritas.

ERW - Eletric Resistance Welding .

ETs - Espécimes Tubulares.

IW - Induction Welding.

LF - Linha de Fusão.

MIG - Métodos de soldagem a arco elétrico que utilizam gases inertes ou uma mistura destes para a proteção da poça de fusão.

MAG - Métodos de soldagem a arco elétrico que utilizam gases ativos ou uma mistura de gases ativos e inertes para a proteção da poça de fusão. PETROBRAS - Petróleo Brasileiro S/A.

SMTS - Specified Minimum Tensile Strength (Limite Mínimo de Tração Especificado).

SMYS - Specified Minimum Yield Strength (Limite Mínimo de Escoamento Especificado).

SAW - Submerged Arc Welding (Solda por Arco Submerso)

TIG - Método de soldagem a arco elétrico que utiliza eletrodo de tungstênio e um gás inerte para a proteção da poça de fusão.

TH - Teste Hidrostático.

TG - Tamanho de Grão ASTM.

TRANSPETRO - Empresa Subsidiária da PETROBRAS, responsável pela operação de dutos e terminais.

TT - Temperatura de Transição.

UOE - Processo de conformação de tubos com costura longitudinal a partir de chapa bobinada.

ZTA - Zona Termicamente Afetada. 